\title{
Cancer Patients and Oncology Clinical Practice in COVID-19 Pandemic
}

Editorial

\author{
Evangelos Karamitrousis ${ }^{1}$, Michalis Liontos $^{2}$, Nikolaos Tsoukalas $^{3 *}$ \\ ${ }^{1}$ Department of Medical Oncology, Democritus University of Thrace, Alexandroupolis, Greece \\ ${ }^{2}$ Department of Clinical Therapeutics, Alexandra Hospital, Athens, Greece \\ ${ }^{3}$ Department of Oncology, 401 General Military Hospital, Athens, Greece
}

Since early 2020, we are dealing with the unexpected outspread of COVID-19 pandemic, at a worldwide scale. COVID-19 is an infection from a novel virus from corona family (Severe Acute Respiratory Syndrome Corona Virus 2 - SARS-CoV-2) that was first reported in December 2019 in China (Wuhan, Hubei province).

One of the most prominent characteristics of COVID-19 is the rapid spreading, with more than 20 million cases and more than 700,000 deaths all-over the world by mid August 2020. COVID-19 symptoms are in most cases of respiratory origin, including fever, cough, chest pain, and shortness of breath. ${ }^{[1]}$ However, SARS-CoV-2 could also affect any system of the human body and present with a variety of symptoms, such as gastrointestinal or ocular ones. ${ }^{[1]}$ Currently, there are neither vaccines, nor specific drugs against SARS-CoV-2 and therapy of the infection is mainly symptomatic.

Venous thromboembolism (VTE) is common in acutely ill patients with COVID-19 infection, seen in up to $1 / 3$ of patients in the intensive care unit (ICU), even when prophylactic anticoagulation is used. ${ }^{[2]}$ The most common pattern of coagulopathy observed in patients hospitalized with COVID-19 is characterized by elevations in fibrinogen and D-dimer levels. This correlates with parallel rise in inflammatory markers. [2] The management of VTE in COVID-19 patients is based in anticoagulation therapy mainly using Low Molecular Weight Heparins (LMWHs). ${ }^{[2]}$ Besides that, thromboprophylaxis in COVID-19 patients should be offered on a case by case approach. Moreover, heparin effects beyond anticoagulation could play a role.

Cancer patients are known to be immunocompromised due to cancer itself and the antineoplastic treatment. Thus, cancer patients are labeled as "COVID-19 vulnerable".
[3] Due to the evolving situation, no definitive data exist describing the effect of COVID-19 in cancer patients. Accumulating evidence though suggest that cancer patients are at higher risk of SARS-Cov-2 infection and have increased mortality and morbidity from COVID-19. [4,5] Analogous evidence led to a dramatic change in cancer patients' management amid the initial eruption of the pandemic such as interruption of chemotherapies, change of intravenous treatments to oral regimens as well as change in the frequency of immunotherapies. This is applicable to specific subgroups of patients. For example, patients with thoracic malignancies are especially vulnerable to COVID-19 due to comorbidities, smoking, and disease related lung damage. In this issue, optimal management of patients with rare lung cancer histologies as well as the clinical biomarkers to guide treatment in lung cancer patients are reviewed. ${ }^{[6,7]}$ In addition, Li et al. review the data regarding the role of chemotherapy and radiotherapy in patients near their end of life. ${ }^{[8]}$ Currently, all major oncological associations have published guidelines to guide management of cancer patients during COVID-19 pandemic that prioritize diagnostic procedures, surgical, and medical treatment in relation to the anticipated benefit and the risk of SARS-CoV-2 infection.

In addition, cancer, alike COVID-19 infection, is strongly associated with thrombosis. Venous Thromboembolism (VTE) occurs in up to $20 \%$ of patients with cancer, contributes significantly in morbidity and mortality, and interferes with cancer treatment. In fact, Cancer-Associated Thrombosis (CAT) is the second leading cause of death among cancer patients. Moreover, oncology patients have higher rates of VTE recurrence and bleedings with anticoagulants. Finally, CAT can be 
the first clinical manifestation of an undiagnosed cancer. Consequently, VTE prophylaxis or treatment is of great importance for cancer patients, even more for those with COVID-19 infection. Currently, Low Molecular Weight Heparins (LMWHs) constitute the established anticoagulation treatment in cancer patients, but compelling data for novel agents emerge. Evidence regarding all available treatment options for VTE treatment and prophylaxis in cancer patients is reviewed in this issue of $\mathrm{FCO} .^{[9]}$ Since cancer patients may also tolerate higher doses of LMWHs without increasing the bleeding risk further, a high thrombotic burden-adapted strategy could help high-risk patients who may benefit from the use of more effective LMWHs doses if they have low bleeding risk. ${ }^{[10]}$

It is of great importance, that doctors and caregivers pay attention in this vulnerable group of patients. There is a great need for continuous care in cancer

\section{References}

[1] Guan WJ, Ni ZY, Hu Y, Liang WH, Ou CQ, $\mathrm{He}$ JX, et al; China Medical Treatment Expert Group for Covid-19. Clinical Characteristics of Coronavirus Disease 2019 in China. N Engl J Med. 2020;382(18): 1708-1720.

[2] Al-Samkari $H$, Karp Leaf RS, Dzik WH, Carlson JCT, Fogerty AE, Waheed $A$, et al; COVID-19 and coagulation: bleeding and thrombotic manifestations of SARS-CoV-2 infection. Blood. 2020 Jul 23;136(4):489-500.

[3] G. Curigliano, S. Banerjee, A. Cervantes, M. Garassino, P. Garrido, N. Girard, et al; Managing cancer patients during the COVID-19 pandemic: An ESMO Interdisciplinary Expert Consensus. Annals of Oncology, https://www.annalsofoncology.org/ article/S0923-7534(20)39948-8/pdf.

[4] Desai A, Sachdeva S, Parekh T, Desai R. COVID-19 and Cancer: Lessons From a Pooled Meta-Analysis.JCO Glob Oncol. 2020 Apr;6:557559.

[5] Emami A, Javanmardi F, Pirbonyeh N, Akbari A. Prevalence of Underlying Diseases in Hospitalized Patients with COVID-19: a Systematic Review and Meta-Analysis. Arch Acad Emerg Med. 2020 Mar 24;8(1):e35. eCollection 2020.

[6] Ioannidis G. Pulmonary Large-Cell Neuroendocrine Carcinoma: Therapeutic Challenges and Opportunities. Forum ClinOncol 2020 11(1), 7-21. patients, both in diagnosis and therapy, despite the potential exposure in SARS-CoV-2 infection, which could be fatal for them. Doctors should make efforts, in order to protect cancer patients from the infection, together with the disease progression resulting from any diagnostic and therapeutic delays. Continuation of active anticancer therapy should be individualized according to the patient's condition, the purpose of treatment, tolerance, and response to treatment. In addition, cancer and its therapy make these patients more susceptible to respiratory infections, due to the compromised immune response to respiratory bacteria and viruses. Thus, the epidemic spread of COVID-19 has posed great challenges to the clinical practice of oncologists, especially for integrative cancer therapy.

[7] Ntanasis-Stathopoulos I, Kyriazoglou A, Dimopoulos MA, Gavriatopoulou M. Clinical biomarkers directing the management of patients with colon and lung cancer (beyond oncogeneaddicted NSCLC). Forum ClinOncol 2020 11(1), 3-6.

[8] Li B, Perkins S, Phillips S, Martin S, Hsieh S, Shinohara E, et al; A Review of Chemotherapy and Radiotherapy Near the End of Life in Individuals with Metastatic Non-small Cell Lung Cancer. Forum ClinOncol 2020 11(1), 29-36.

[9] Savvari P. Cancer-associated thromboembolism: could direct-acting oral anticoagulants be a promising therapeutic option? Literature review. Forum ClinOncol 2020 11(1), 22-28.

[10] Tsoukalas N, Papakotoulas P, Christopoulou A, Ardavanis A, Koumakis G, Papandreou C, et al; Real-World Data on Thromboprophylaxis in Active Cancer Patients: Where Are We? Are We Getting There? Cancers (Basel). 2020 Jul 15;12(7):E1907. 